\title{
A network pharmacology approach to explore mechanisms of Buyanghuanwu decoction for treatment of cerebral infarction
}

\author{
Yunyao Jiang, Nan Liu, Jianxun Liu \\ Institute of Basic Medical Sciences, Xiyuan hospital, China Academy of Chinese Medical Sciences, China
}

Cerebral infarction $(\mathrm{CI})$, is a type of ischemic stroke, results from a blockage of the blood flow through the vessels providing blood supply to functional regions in the brain, and can lead to many results including a loss of physiologic functions, paralysis, and even death. The point of this study is to explore and investigate mechanisms of Buyanghuanwu decoction for treatment of CI using a network pharmacology approach.

TCMSP database, drugbank database and PharmMapper Server were used and combined with oral bioavailability and drug analysis to screen the components of Buyanghuanwu decoction and predict the potential targets. Then, Cytoscape 3.5.1 software was used to construct compounds-targets network and the protein-protein interaction (PPI) networks for targets of compounds and CI-related targets and merge the two PPI networks to acquire active targets. Gene ontology (GO) and KEGG pathway analysis of active targets were carried out by DAVID online analysis tool and KOBAS 3.0 software.

In total of 150 screened compounds and 232 potential targets of Buyanghuanwu decoction were obtained. And 207 active targets that Buyanghuanwu decoction treated $\mathrm{CI}$ were determined by the merged PPI network. Results of GO and KEGG pathway analysis indicated that Buyanghuanwu decoction might have a role in treating CI by regulating some biological processes including apoptotic process, gene silencing, and cell adhesion, and involving some molecular function, such as protein binding, enzyme binding, and transcription factor binding. And the top 20 significantly enriched pathways, such as neurotrophin signaling pathway, cell cycle, PI3K-Akt signaling pathway, and estrogen signaling pathway were shown.

In conclusion, the mechanisms of Buyanghuanwu decoction for treatment of $\mathrm{CI}$ might be concerned with neurotrophin signaling pathway, PI3K-Akt signaling pathway, MAPK signaling pathway, focal adhesion, and estrogen signaling pathway. Among them, the regulation of PI3K-Akt signaling pathway might be one of the most crucial mechanisms. 